\title{
Origins and Breadth of the Theory of Higher Homotopies
}

\author{
J. Huebschmann ${ }^{1}$ \\ ${ }^{1}$ USTL, UFR de Mathématiques \\ CNRS-UMR 8524 \\ 59655 Villeneuve d'Ascq Cédex, France \\ Johannes.Huebschmann@math.univ-lille1.fr
}

October 27, 2018

\begin{abstract}
Higher homotopies are nowadays playing a prominent role in mathematics as well as in certain branches of theoretical physics. The purpose of the talk is to recall some of the connections between the past and the present developments. Higher homotopies were isolated within algebraic topology at least as far back as the 1940's. Prompted by the failure of the Alexander-Whitney multiplication of cocycles to be commutative, Steenrod developed certain operations which measure this failure in a coherent manner. Dold and Lashof extended Milnor's classifying space construction to associative $H$-spaces, and a careful examination of this extension led Stasheff to the discovery of $A_{n}$-spaces and $A_{\infty}$-spaces as notions which control the failure of associativity in a coherent way so that the classifying space construction can still be pushed through.

Algebraic versions of higher homotopies have, as we all know, led Kontsevich eventually to the proof of the formality conjecture. Homological perturbation theory (HPT), in a simple form first isolated by Eilenberg and Mac Lane in the early 1950's, has nowadays become a standard tool to handle algebraic incarnations of higher homotopies. A basic observation is that higher homotopy structures behave much better relative to homotopy than strict structures, and HPT enables one to exploit this observation in various concrete situations which, in particular, leads to the effective calculation of various invariants which are otherwise intractable.

Higher homotopies abound but they are rarely recognized explicitly and their significance is hardly understood; at times, their appearance might at first glance even come as a surprise, for example in the Kodaira-Spencer approach to deformations of complex manifolds or in the theory of foliations.
\end{abstract}




\section{Contents}

1 Introduction $\quad 2$

2 The formality conjecture

3 Early History

4 Various 20'th century higher homotopies

5 Homological perturbations $\quad 7$

6 Quantum groups $\quad 8$

7 Operads

8 Deformation theory

9 Strings

10 Cohomological physics

11 Higher homotopies, homological perturbations, and the working mathematician

\section{Introduction}

It gives me great pleasure to join in this celebration of Murray Gerstenhaber's 80'th and Jim Stasheff's 70'th birthday. I had the good fortune to get into contact with Jim some 25 years ago. In 1981/82 I spent six months at the Swiss Federal Institute of Technology (Zürich) as a Research Scholar. At the time, I received a letter from Jim asking for details concerning my application of twisting cochains to the calculation of certain group cohomology groups. What had happened? At Zürich, I had lectured on this topic, and Peter Hilton was among the audience. This was before the advent of the internet; not even e-mail was available, and people would still write ordinary snail mail letters. Peter Hilton travelled a lot and in this way transmitted information; in particular, he had told Jim about my attempts to do these calculations by means of twisting cochains. By the way, since Peter Hilton was moving around some much, once someone tried to get hold of him, could not manage to do so, and asked a colleague for advice. The answer was: Stay where you are, and Peter will certainly pass by.

At that time I knew very little about higher homotopies, but over the years I have, like many of us, learned much from Jim's insight, his habit of bringing his readers, students, and coworkers out from "behind the cloud of unknowing", to quote some of Jim's own prose in his thesis. All of us have benefited from Jim's generosity with ideas.

I cannot reminisce indefinitely, yet I would like to make two more remarks, one related with language and in particular with language skills: For example, I vividly remember, in 
the fall of 1987, there was a crash at Wall Street. I inquired via e-mail-which was then available-, whether this crash created a problem, for Jim or more generally for academic life. His answer sounded somewhat like "Not a problem, but quite a tizzy here". So I had to look up the meaning of "tizzy" in the dictionary. This is just one instance of how I and presumably many others profitted from Jim's language skills. Sometimes Jim answers an e-mail message of mine in Yiddish-apparently his grandfather spoke Yiddish to his father. There is no standard Yiddish spelling and, when I receive such a message, to uncover it, I must read it aloud myself to understand the meaning, for example "OY VEH" which, in standard German spelling would be "Oh Weh".

I feel honoured by the privilege to have been invited to deliver this tribute talk. I would like to make a few remarks related to Murray Gerstenhaber. I have met Murray some 20 years ago when I spent some time at the Institute in Princeton. From my recollections, Murray was then a member of the alumni board of the Institute and was always very busy. We got into real scientific and personal contact only later. In particular, I was involved in reviewing some of the Gerstenhaber-Schack results, and I will never forget that I learnt from Murray about Wigner's approach to the idea of contraction. Also from time to time, beyond talking about mathematics, we talked about history. For example, Ruth Gerstenhaber once observed how people would gather for tea in the Fuld Hall common room in the afternoon as usual around the table, and no-one would say a word but, one after another, would eventually leave the room murmuring "There is no counter-example." The perception of a mathematician through a non-mathematician is sometimes revealing.

Before I go into the mathematical details of my talk, let us wish many more years to Jim and Murray and their wives.

Let me now turn to my talk. There would be much more to say than what I can explain in the remaining time. I shall touch on various topics and make a number of deliberate choices and I will make the attempt to explain some pieces of mathematics. However, my exposition will be far from being complete or systematic and will unavoidably be biased. For example there are higher homotopies traditions in Russia and in Japan related with Lie loops, Lie triple systems and the like which I cannot even mention, cf. e. g. [32] and [42]. There is a good account of Jim Stasheff's contributions up to his 60'th birthday, published at the occasion of this event [38]. This was just before the advent of Kontsevich's proof of the formality conjecture. I will try to complement this account and can thereby, perhaps, manage to avoid too many repetitions. Also I will try to do justice to a number of less well known developments.

\section{The formality conjecture}

Let me run right into modern times and right into our topic: Algebraic versions of higher homotopies have, as we all know, led Kontsevich eventually to the proof of the formality conjecture [34]: Let $M$ be a smooth manifold, let $A=C^{\infty}(M)$ and $L=\operatorname{Vect}(M)$, and consider the exterior $A$-algebra $\Lambda_{A} L$ on $L$. Let Hoch $(A)$ denote the Hochschild complex of $A$, suitably defined, e. g. in the Fréchet sense. Given the vector fields $X_{1}, \ldots, X_{n}$ on 
$M$, let $\Phi_{X_{1}, \ldots, X_{n}}$ be the Hochschild cochain given by

$$
\Phi_{X_{1}, \ldots, X_{n}}\left(a_{1}, \ldots, a_{n}\right)=\frac{1}{n !} \sum \operatorname{sign}(\sigma) \prod_{j=1}^{n} X_{\sigma(j)}\left(a_{j}\right), \quad a_{1}, \ldots a_{n} \in A
$$

By a version of a classical result of Hochschild-Kostant-Rosenberg, the obvious map

$$
\Lambda_{A} L \longrightarrow \operatorname{Hoch}(A), \quad X_{1} \wedge \ldots \wedge X_{n} \mapsto \Phi_{X_{1}, \ldots, X_{n}},
$$

is an isomorphism on cohomology. That is to say, the Hochschild cohomology of $A=$ $C^{\infty}(M)$ amounts to the graded algebra $\Lambda_{A} L$ of multi vector fields on $M$.

The standard Schouten-Nijenhuis bracket $[\cdot, \cdot]$ turns the suspension $s\left(\Lambda_{A} L\right)$ of $\Lambda_{A} L-$ this is $\Lambda_{A} L$, regraded up by 1 , into an ordinary graded Lie algebra. Here the grading convention is the standard one in algebraic topology to the effect that, in particular, a differential lowers degree by 1 . Likewise, the familiar Gerstenhaber bracket on $\operatorname{Hoch}(A)$ turns the suspension $s(\operatorname{Hoch}(A))$ of $\operatorname{Hoch}(A)$ into an ordinary differential graded Lie algebra. However, the morphism (2.1), while certainly being compatible with the differentials, is not compatible with the Lie brackets.

For any differential graded Lie algebra $\mathfrak{g}$, the familiar $\mathrm{C}$ (artan) $\mathrm{C}$ (hevalley) E(ilenberg)construction $\mathrm{S}^{\prime}[\mathfrak{g}]$ furnishes a d(ifferential) $\mathrm{g}$ (raded) coalgebra. In fact, given $\mathfrak{g}$, differential graded Lie algebra structures on $\mathfrak{g}$ can be characterized in terms of dg coalgebra structures on the symmetric coalgebra $\mathrm{S}^{\prime}[s(\mathfrak{g})]$ on the suspension $s(\mathfrak{g})$ of $\mathfrak{g}$ : They correspond precisely to the dg coalgebra structures determined by a linear term, the differential, and a quadratic term, the bracket. This allows for immediate generalization: An sh-Lie algebra is a vector space $\mathfrak{g}$ together with a coalgebra differential on the symmetric coalgebra $S^{\prime}[s(\mathfrak{g})]$ on the suspension $s(\mathfrak{g})$ of $\mathfrak{g}$. The formality conjecture, as formulated and established by Kontsevich [34], says that (2.1) extends to a Lie algebra twisting cochain

$$
\tau: \mathrm{S}^{\prime}\left[s^{2}\left(\Lambda_{A} L\right)\right] \longrightarrow s(\operatorname{Hoch}(A)) .
$$

Here $\tau$ being a twisting cochain means that $\tau$ satisfies the deformation or Maurer-Cartan equation. Such a Lie algebra twisting cochain furnishes an sh-map from the ordinary (differential) graded Lie algebra $s\left(\Lambda_{A} L\right)$ to the ordinary differential graded Lie algebra $s(\operatorname{Hoch}(A))$.

The twisting cochain $\tau$ has homogeneous constituents $\tau_{j}, \tau_{1}$ being essentially the above morphism (2.1). The higher terms $\tau_{j}(j \geq 2)$ are an instance of higher homotopies, and $\tau$ is an instance of an sh-map, a term created by Jim Stasheff, inspired by terminology introduced by Sugarawa [56], see Section 4 below; here "sh" stands for "strongly homotopic". Thus, without having the language and notation of higher homotopies and that of deformations at his disposal-remarkably, both Murray Gerstenhaber and Jim Stasheff are behind the scene at this point and both from 1963-, Kontsevich would not even have been able to phrase the formality conjecture. This confirms a variant of an observation which, with a grain of salt, reads thus: Mathematics consists in continuous and discreet development of language and notation.

A key observation, advocated by Jim Stasheff from early on, is this: Even though we start with strict objects, an sh-map between them may lead to new insight, not necessarily available from ordinary strict maps. This kind of observation has been successfully 
exploited in rational homotopy theory for decades. Kontsevich noticed its significance in an area at first independent of rational homotopy and, furthermore, managed to exhibit a particular sh-map which establishes the formality conjecture.

$\mathrm{R}$. Thom had raised the issue of existence of a graded commutative differential graded algebra of cochains on a space [58]. This prompted the development of rational homotopy, starting notably with D. Quillen [41] and D. Sullivan [53]. A space whose rational (or real) cochain algebra is sh-equivalent to its cohomology algebra is said to be formal, the term formal referring to the fact that the rational homotopy type is then a formal consequence of the structure of the cohomology ring. The term formality conjecture derives from this tradition.

The statement of the formality conjecture implies, as we know, that every Poisson bracket on a smooth manifold admits a deformation quantization.

\section{Early History}

One of the origins of homotopy is Gauß' analytic expression for the linking number of two closed curves (1833). One of the origins of higher homotopies is the idea of a classifying space; this idea goes again back to Gauß (1828). Another origin of higher homotopies is the usage of resolutions. It is a common belief, perhaps, that resolutions go back at least to Hilbert's exploration of syzygies [17]. Hilbert studied syzygies in order to show that the generating function for the number of invariants of each degree is a rational function. He also showed that, for a homogeneous ideal $I$ of a polynomial ring $S$, the "number of independent linear conditions for a form of degree $d$ in $S$ to lie in $I$ " is a polynomial function of $d$. However, this is not the entire story. The problem of counting the number of conditions had already been considered for some time; it arose both in projective geometry and in invariant theory. A general statement of the problem, with a clear understanding of the role of syzygies-but without the word, introduced a few years later by Sylvester (1814-1897) [57]-is given by Cayley (1821-1895) [3]. In fact, in a sense, Cayley somewhat develops what is nowadays referred to as the Koszul resolution [35] more than 100 years before Koszul. The terminology homotopy was apparently created by $\mathrm{H}$. Poincaré (1895). Poincaré also introduced the familiar loop composition. Thus we see that, in the historical perspective, Jim Stasheff is in excellent company.

\section{Various 20'th century higher homotopies}

Prompted by the failure of the Alexander-Whitney multiplication of cocycles to be commutative, Steenrod developed the system of $\cup_{i}$-products [54]. These induce the squaring operations which, in turn, measure this failure of commutativity in a coherent manner. The non-triviality of these operations implies in particular that, over the integers, there is no way to introduce a differential graded commutative algebra of cochains on a space. The $\cup_{i}$-products entailed the development of $\mathrm{s}$ (trongly)h(omotopy)c(ommutative) structures as well as that of Steenrod operations.

An $A_{\infty}$-structure may be described as a system of higher homotopies together with suitable coherence conditions. Massey products [37] may be seen as invariants of cer- 
tain $A_{\infty}$-structures. An elementary example arises from the familiar Borromean rings, consisting of three circles which are pairwise unlinked but all together are linked. The name "Borromean" derives from their appearance in the coat of arms of the house of the aristocratic Borromean family in Northern Italy. If we regard these rings as situated in the 3-sphere, then the cohomology ring of the complement is a trivial algebra, but there is a Massey product of three variables detecting the simultaneous linking of all three circles.

At the time Massey products were isolated, Jim Stasheff was a graduate student at Princeton. His advisor J. Moore suggested he look at the problem of determining when a cohomology class of a based loop space $\Omega X$ was a suspension or a loop class, i. e. came from a cohomology class of $X$. In pursuing this question, Stasheff was led to work of Sugawara [55], who had a recognition principle for characterizing loop spaces up to homotopy type.

The ordinary loop multiplication on $\Omega X$ gives it the structure of an H-space that is associative up to homotopy. Moore's version of the loop space shows that there is a based loop space which is homotopic to the familiar one for which the loop multiplication is strictly associative. The conclusion is that associativity is not a homotopy invariant property; we owe Jim a complete understanding of the homotopy invariance properties of associativity, and his solution furnishes a clean recognition principle for loop spaces and, in fact, for an entire hierarchy of spaces between loop spaces and H-spaces, the loop spaces being spaces which admit a classifying space.

Specifically, Stasheff defined a nested sequence of homotopy associativity conditions and called a space an $A_{n}$-space if it satisfies the $n$ 'th condition. Every space is an $A_{1^{-}}$ space, an $\mathrm{H}$-space is an $A_{2}$-space, and every homotopy associative $\mathrm{H}$-space is $A_{3}$. An $A_{\infty}$-space has the homotopy type of a loop space.

A. Dold and R. Lashof [4] generalized to associative H-spaces Milnor's construction of a classifying space for a topological group [40]. Jim Stasheff extended the Dold-Lashof construction to $A_{\infty}$-spaces through his study of homotopy associativity of higher order: an $A_{\infty}$-structure precisely gives a classifying space. All this was worked out in his thesis, published as [45]. Sugawara had introduced conditions for a group-like space, see the definition in terms of the conditions 3.1-3.3 on p. 129 of [55] to be imposed upon two maps related by what Sugawara had called an iteration of the standard relations. Altering the appropriate part of these conditions to suit the case of associativity more precisely and naturally led Jim Stasheff, apparently prompted by F. Adams, to isolating a now familiar family of polyhedra, that of associahedra. We shall see below that these polyhedra actually constitute an operad. Moreover, following Sugawara [56], Stasheff defined maps of $A_{n^{-}}$ spaces, referred to as $A_{n}$-maps, which are special kinds of $H$-maps [45] (Def. 4.4 p. 298); these maps are homotopy multiplicative in a strong sense. Via Sugawara's work, $A_{n}$-maps are related to the Dold and Lashof construction. When the homotopies defining an $A_{n^{-}}$ map exist for all $n$, the corresponding map is strongly homotopy multiplicative in the sense of Sugawara [56] (p. 259). Thus the sh-terminology we are so familiar with nowadays was born.

The algebraic analogue of an $A_{n}$-space in the category of algebras is an $A_{n}$-algebra, the case $n=\infty$ being included here. The original and motivating example was provided by the singular chains on the based loop space of a space. This notion, and variants thereof, has found many applications. One such variant, $L_{\infty}$-algebras, have already been 
mentioned. A key observation here is that $A_{\infty}$-structures behave correctly with respect to homotopy, which is not the case for strict structures. What corresponds to the classifying space construction in geometry is now the bar tilde construction. Inside the bar tilde construction, Massey products show up which determine the differentials in the resulting bar construction spectral sequence. Stasheff referred to these operations as Yessam operations. History relates that once, at the end of a talk of Jim's, S. Mac Lane asked the question: Who was Yessam?

Let me recall a warning, one of Jim's favorite warnings in this context: When the differential of an $A_{\infty}$-algebra is zero, the conditions force the algebra to be strictly associative but there may still be non-trivial higher operations encapsulating additional information, as the example of the Borromean rings already shows where the non-triviality of the Massey product reflects the triple linking.

Jim Stasheff continued to work in the realm of fibrations. There is, for example, a notion of topological parallel transport developed by him. A recent joint article of J. Stasheff and J. Wirth entitled Homotopy transition cocycles [52] reworks and extends J. Wirth's thesis written in 1965 under the supervision of J. Stasheff.

\section{$5 \quad$ Homological perturbations}

Homological perturbation theory (HPT) has nowadays become a standard tool to construct and handle $A_{\infty}$-structures. The term "homological perturbation" is apparently due to J. Milgram. The basic HPT-notion, that of contraction, was introduced in Section 12 of [5]: A contraction

$$
(X \underset{\pi}{\stackrel{\nabla}{\rightleftarrows}} Y, h)
$$

consists of chain complexes $X$ and $Y$, chain maps $\nabla: X \rightarrow Y$ and $\pi: Y \rightarrow X$, and a degree 1 morphism $h: Y \rightarrow Y$ such that

$$
\pi \nabla=\mathrm{Id}, \nabla \pi-\mathrm{Id}=d h+h d, h \nabla=0, \pi h=0, h h=0 .
$$

The notion of "recursive structure of triangular complexes" in Section 5 [16] is also an example of what was later identified as a perturbation. The "perturbation lemma" is lurking behind the formulas in Chapter II of Section 1 of [44] and seems to have first been made explicit by M. Barrat (unpublished). The first instance known to us where it appeared in print is [2]. Jim Stasheff collaborated with various colleagues on questions related with homological perturbation theory [12], [13], [14] including myself [31]. An issue dealt with in these papers, as well as in my joint paper [30] with T. Kadeishvili, is that of compatibility of the perturbation constructions with algebraic structure. This issue actually shows up when one tries to construct e. g. models for differential graded algebras.

A homological algebra and higher homotopies tradition was created as well by Berikashvili and his students in Georgia (at the time part of the USSR). More precise comments about the historical development until the mid eighties may be found in the article [30], and some specific comments about the Georgian tradition in [23]. 
In the articles [18], [19], [20], I explored the compatibility of the perturbation constructions with algebraic structure and developed suitable algebraic HPT-constructions to exploit $A_{\infty}$-modules arising in group cohomology. In this vein, I constructed suitable free resolutions from which I was able to do explicit numerical calculations in group cohomology which until today still cannot be done by other methods. In particular, spectral sequences show up which do not collapse from $E_{2}$. These results illustrate a typical phenomenon: Whenever a spectral sequence arises from a certain mathematical structure, there is, perhaps, a certain strong homotopy structure lurking behind, and the spectral sequence is an invariant thereof. The higher homotopy structure is then somewhat finer than the spectral sequence itself.

\section{Quantum groups}

The issues of associativity and coassociativity, as clarified by Jim Stasheff, play a major role in the theory of quantum groups and variants thereof, e. g. quasi-Hopf algebras. Suffice it to mention here that Drinfel'd has introduced a notion of quasi-Hopf algebra in which coassociativity of the diagonal is modified in a way in which the pentagon condition plays a dominant role, analogous to the hexagonal Yang-Baxter equation replacement for cocommutativity. Now, given a quasi-Hopf algebra $A$, the quasi-Hopf structure induces a multiplication $\mathrm{BC} \times \mathrm{BC} \longrightarrow \mathrm{BC}$ on the classifying space $\mathrm{BC}$ of the category $\mathcal{C}$ of $A$ modules, and the quasi-Hopficity says that this multiplication is homotopy associative. More details and suitable references may be found in [47] and [48].

\section{Operads}

The notion of $A_{n}$-spaces and the clarity they provide for the recognition problem for topological groups became the basis for the development of homotopy invariant algebraic structures. In particular, the recognition problem for infinite loop spaces and the simultaneous interest in coherence properties in categories led to the idea of an operad [36], [39]. With hindsight we recognize that a space is an $A_{\infty}$-space if and only if it is an algebra over a suitably defined operad, the non-symmetric operad $\mathcal{K}=\left\{K_{n}\right\}$ of associahedra. In fact, this is the main result of Stasheff's thesis, though not spelled out in this language:

A connected space $Y$ of the homotopy type of a $C W$-complex has the homotopy type of a loop space if and only if there exist maps $K_{n} \times Y^{n} \rightarrow Y$ which fit together to make $Y$ an algebra over the operad $\mathcal{K}$. In fact, $Y$ then has the homotopy type of the space $\Omega X$ where $X$ is constructed as a quotient of $\coprod K_{n} \times Y^{n}$. This brings the generalized classifying space construction to the fore.

Likewise a graded object is an $A_{\infty}$-algebra if and only if it is an algebra over a suitably defined operad, and an $L_{\infty}$-algebra can be characterized in the same manner as well. In recent years many more new phenomena and structures and, in particular, applications of operads have been found, in particular in the theory of moduli spaces and in mathematical physics. 


\section{Deformation theory}

There is an obvious formal relationship between homological perturbations and deformation theory but the relationship is actually much more profound: In [15], Steve Halperin and Jim Stasheff developed a procedure by means of which the classification of rational homotopy equivalences inducing a fixed cohomology algebra isomorphism can be achieved. Moreover, one can explore the rational homotopy types with a fixed cohomology algebra by studying perturbations of a free differential graded commutative model by means of techniques from deformation theory. This was initiated by M. Schlessinger and J. Stasheff [43]. A related and independent development, phrased in terms of what is called the functor $\mathcal{D}$, is due to N. Berikashvili and his students at Georgia, notably T. Kadeishvili

and S. Saneblidze. Some details and references are given in [23]. A third approach in which only the underlying graded vector space was fixed is due to Y. Felix [7].

More recently, prompted by a paper of Baranikov and Kontsevich [1], Jim Stasheff and I developed an approach to constructing solutions of the master equation by means of techniques from HPT [31]. In that paper, we restricted attention to contractions of a differential graded Lie algebra onto its homology. More recently, I extended this approach to the situation of a contraction of a differential graded Lie algebra onto a general chain complex and thereby established the perturbation lemma for differential graded Lie algebras [28]. Further, I generalized the statement of the perturbation lemma to arbitrary sh-Lie algebras [29].

\section{Strings}

Operads and sh-Lie algebras show up naturally in string and conformal field theories, and Jim Stasheff contributed to this area as well. Some details and more references may be found in [51].

\section{Cohomological physics}

One of Jim's long-term interests is physics. Due to his efforts it is, perhaps, no longer a surprise that some structures of interest in physics can be explored by means of tools going back to topology, including graded Lie algebras and homological perturbations. Jim contributed to anomalies [46] and invested time and effort to unravel, for example, the structure behind a field theory construction which originally goes back to Batalin, Fradkin, and Vilkovisky. The term "cohomological physics" was created by Jim. See in particular [49] and [50] for details.

\section{Higher homotopies, homological perturbations, and the working mathematician}

I have already explained how higher homotopies and homological perturbations may be used to solve problems phrased in language entirely different from that of higher homo- 
topies and HPT. Higher homotopies and HPT-constructions occur implicitly in a number of other situations in ordinary mathematics where they are at first not even visible. I can only mention some examples; these are certainly not exhaustive.

- Kodaira-Nirenberg-Spencer: Deformations of complex structures [33];

- Frölicher spectral sequence of a complex manifold [22; 24];

- Toledo-Tong: Parametrix [59];

- Fedosov: Deformation quantization [6];

- Whitney, Gugenheim: Extension of geometric integration to a contraction [9], 60]. Whitney's geometric integration theory laid some of the ground work for Sullivan's theory of rational differential forms quoted above. The upshot of Gugenheim's contribution here is that the integration map in de Rham theory is sh-multiplicative, the de Rham algebra being an ordinary graded commutative algebra. This situation is formally the same as that of the formality conjecture explained above.

- Huebschmann: Foliations [25]; in this paper, the requisite higher homotopies are described in terms of a generalized Maurer-Cartan algebra.

- Huebschmann: Equivariant cohomology and Koszul duality [26], [27].

- Operads; see e. g. the conference proceedings which contain the article [51].

\section{References}

[1] S. Barannikov and M. Kontsevich, Frobenius manifolds and formality of Lie algebras of polyvector fields, Internat. Math. Res. Notices 4 (1998), 201-215, alg-geom/9710032.

[2] R. Brown, The twisted Eilenberg-Zilber theorem, Celebrazioni Archimedee del Secolo XX, Simposio di topologia 1964.

[3] A. Cayley, On the Theory of Elimination, Cambridge and Dublin Math. Journal 3 (1848), 116-120.

[4] A. Dold and R. Lashof, Principal quasi-fibrations and fiber homotopy equivalence of bundles, Illinois J. of Math. 3 (1959), 285-305.

[5] S. Eilenberg, S. MacLane, On the groups $H(\pi, n)$. I, Ann. of Math. 58 (1953), 55-106, II. Methods of computation, Ann. of Math. 60 (1954), 49-139.

[6] B. V. Fedosov, A simple geometrical construction of deformation quantizations, J. Diff. Geom. 40 (1994), 213-238.

[7] Y. Felix, Classification homotopique des espaces rationnels de cohomologie donnée, (French). Bull. Soc. Math. Belg. Sér. B 31 (1979), 75-86. 
[8] V.K.A.M. Gugenheim: On the chain complex of a fibration, Illinois J. of Math. 16 (1972), 398-414.

[9] V.K.A.M. Gugenheim, On the multiplicative structure of the de Rham theory, J. of Diff. Geom. 11 (1976), 309-314.

[10] V.K.A.M. Gugenheim: On a perturbation theory for the homology of the loop space, J. of Pure and Applied Algebra 25 (1982), 197-205.

[11] V.K.A.M. Gugenheim and R. J. Milgram: On successive approximations in homological algebra, Trans. Amer. Math. Soc. 150 (1970), 157-182.

[12] V.K.A.M. Gugenheim and J. D. Stasheff, On perturbations and $A_{\infty}$-structures, Festschrift in honor of G. Hirsch's 60'th birthday, ed. L. Lemaire, Bull. Soc. Math. Belgique 38 (1986), 237-245.

[13] V.K.A.M. Gugenheim, L. Lambe and J. D. Stasheff, Algebraic aspects of Chen's twisting cochains, Illinois J. of Math. 34 (1990), 485-502.

[14] V.K.A.M. Gugenheim, L. Lambe and J. D. Stasheff, Perturbation theory in differential homological algebra. II., Illinois J. of Math. 35 (1991), 357-373

[15] S. Halperin and J. D. Stasheff, Obstructions to homotopy equivalences, Advances in Math. 32 (1979), 233-278

[16] A. Heller, Homological resolutions of complexes with operators, Ann. of Math. 60 (1954), 283-303.

[17] D. Hilbert, Über die Theorie der algebraischen Formen, Math. Ann. 36 (1890), 473534 .

[18] J. Huebschmann, Perturbation theory and free resolutions for nilpotent groups of class 2, J. Algebra 126 (1989), 348-399.

[19] J. Huebschmann, Cohomology of nilpotent groups of class 2, J. Algebra 126 (1989), 400-450.

[20] J. Huebschmann, The mod p cohomology rings of metacyclic groups, J. Pure Appl. Algebra 60 (1989), 53-105.

[21] J. Huebschmann, Cohomology of metacyclic groups, Trans. Amer. Math. Soc. 328 (1991), 1-72.

[22] J. Huebschmann, Twilled Lie-Rinehart algebras and differential Batalin-Vilkovisky algebras, math.DG/9811069.

[23] J. Huebschmann, Berikashvili's functor $\mathcal{D}$ and the deformation equation, in: Festschrift in honor of N. Berikashvili's 70th birthday, Proceedings of A. Razmadze Institute 119 (1999), 59-72, math.AT/9906032. 
[24] J. Huebschmann, Differential Batalin-Vilkovisky algebras arising from twilled LieRinehart algebras, Banach Center Publications 51 (2000), 87-102.

[25] J. Huebschmann, Higher homotopies and Maurer-Cartan algebras: quasi-LieRinehart, Gerstenhaber, and Batalin-Vilkovisky algebras, in: The Breadth of Symplectic and Poisson Geometry, Festschrift in Honor of Alan Weinstein, J. Marsden and T. Ratiu, eds., Progress in Mathematics, vol. 232 (2004), 237-302, BirkhäuserVerlag, Boston · Basel · Berlin, math.DG/0311294.

[26] J. Huebschmann, Homological perturbations, equivariant cohomology, and Koszul duality, math.AT/0401160.

[27] J. Huebschmann, Relative homological algebra, homological perturbations, equivariant de Rham theory, and Koszul duality, math. AT/0401161.

[28] J. Huebschmann, The Lie algebra perturbation Lemma, arXiv:0708.3977.

[29] J. Huebschmann, The sh-Lie algebra perturbation Lemma, arXiv:0710.2070.

[30] J. Huebschmann and T. Kadeishvili, Small models for chain algebras, Math. Z. 207 (1991), 245-280.

[31] J. Huebschmann and J. D. Stasheff, Formal solution of the master equation via HPT and deformation theory, Forum mathematicum 14 (2002), 847-868, math.AG/9906036.

[32] M. Kikkawa, Geometry of homogeneous Lie loops, Hiroshima Math. J. 5 (1975), 141-179.

[33] M. Kodaira, L. Nirenberg and D. C. Spencer, On the existence of deformations of complex analytic structures, Ann. of Math. 68 (1958), 450-457.

[34] M. Kontsevich, Deformation quantization of Poisson manifolds, math.QA/9709040.

[35] J. L. Koszul, Homologie et cohomologie des algèbres de Lie, Bull. Soc. Math. France 78 (1950), 65-127.

[36] S. Mac Lane, Categorical Algebra, Bull. Amer. Math. Soc. 75 (1965), 40-106.

[37] W. S. Massey, Some higher order cohomology operations, Symposium internacional de topologia algebraica, Universidad Nacional Autónoma de México and UNESCO, Mexico City, 1958, 145-154.

[38] J. McCleary, An appreciation of the Work of Jim Stasheff, Higher Homotopy Structures in Topology and Mathematical Physics, Proceedings of an International Conference, June 13-15, 1996, at Vassar College, Poughkeepsie, New York, to honor the 60'th birthday of Jim Stasheff, Cont. Math. 227 (1999), 1-16.

[39] P. J. May, The geometry of iterated loop spaces, Lecture Notes in Mathematics 271, Springer-Verlag, Berlin · Heidelberg · New York (1972). 
[40] J. Milnor, Construction of universal bundles. II., Ann. of Math. 63 (1956), 430-436.

[41] D. Quillen, Rational homotopy theory, Ann. of Math. 90 (1969), 205-295

[42] L. V. Sabinin and P. O. Mikheev, On the infinitesimal theory of local analytic loops, Soviet Math. Dokl. 36 (1988), 545-548.

[43] M. Schlessinger and J. Stasheff, Deformation theory and rational homotopy type, preprint 1979; new version July 13, 1998.

[44] W. Shih, Homologie des espaces fibrés, Pub.Math. Sci. IHES 13 (1962)

[45] J. D. Stasheff, Homotopy associativity of H-spaces. I. II. Trans. Amer. Math. Soc. 108 (1963), 275-292; 293-312.

[46] J. D. Stasheff, The de Rham bar construction as a setting for the Zumino, Fadde'ev, etc. descent equations, in: Proceedings of the Symposium on Anomalies, geometry, and Topology, Argonne, Chicago, eds. W. Bardeen, A. White, World Scientific, Singapore (1985).

[47] J. D. Stasheff, Drinfel'd's quasi-Hopf algebras and beyond, in: M. Gerstenhaber and J. Stasheff (eds.), Deformation theory and quantum groups with applications to mathematical physics. AMS-IMS-SIAM 1990 Joint summer research conference, June 14-20, 1990, Amherst, Massachusetts. Cont. Math. 134 (1992), 297-307.

[48] J. D. Stasheff, Differential graded Lie algebras, quasi-Hopf algebras and higher homotopy algebras, in: Quantum groups (Leningrad 1990). Lecture Notes in Mathematics 1510, Springer-Verlag, Berlin (1992), pp. 120-137.

[49] J. D. Stasheff, Deformation theory and the Batalin-Vilkovisky master equation, in: Deformation Theory and Symplectic Geometry, Proceedings of the Ascona meeting, June 1996, D. Sternheimer, J. Rawnsley, S. Gutt, eds., Mathematical Physics Studies, Vol. 20, Kluwer Academic Publishers, Dordrecht/Boston/London (1997), pp. 271284.

[50] J. D. Stasheff, The (secret?) homological algebra of the Batalin-Vilkovisky approach, in: Henneaux, Marc (ed.) et al., Secondary calculus and cohomological physics. Proceedings of a conference, Moscou Russia August 24-31, 1997, Providence RI, AMS, Cont. Math. 219 (1998), 195-210.

[51] J. D. Stasheff, From operads to physically inspired theories, in: L. Loday, J. Stasheff and A. A. Voronov (eds.), Operads, Proceedings of Renaissance conferences, 1995, Hartford (Connecticut)/Luminy (France). Cont. Math. 202 (1997), 53-81.

[52] J. D. Stasheff and J. Wirth, Homotopy transition cocycles, math. AT/0609220.

[53] D. Sullivan, Infinitesimal Computations in Topology, Pub. Math. I. H. E. S 47 (1978), 269-331. 
[54] N. E. Steenrod, Products of cocycles and extensions of mappings, Ann. of Math. 48 (1947), 290-320.

[55] M. Sugawara, On a condition that a space is group-like, Math. J. Okayama Univ. 7 (1957), 123-149.

[56] M. Sugawara, On the homotopy-commutativity of groups and loop spaces, Mem. Coll. Sci. Univ. Kyoto Ser. A Math. 33 (1960/61), 257-269.

[57] J. J. Sylvester, On a Theory of Syzygetic Relations of Two Rational Integral Functions, Comprising an Application of the Theory of Sturm's Functions, and that of the Greatest Algebraic Common Measure, Phil. Trans. Royal Soc. London 143 (1853), 407-548.

[58] R. Thom, Opérations en cohomologie réelle, Séminaire H. Cartan, Exposé 17 $(1954 / 55)$

[59] D. Toledo and Y. L. L. Tong, A parametrix for $\bar{\partial}$ and Riemann-Roch in Čech theory, Topology 15 (1976), 273-301.

[60] H. Whitney, Geometric integration theory, Princeton University Press, Princeton NJ (1957). 